\title{
Temperature-dependent magnetocrystalline anisotropy of rare earth/transition metal permanent magnets from first principles: The light $\operatorname{RCo}_{5}(R=\mathrm{Y}$, La-Gd) intermetallics
}

\author{
Christopher E. Patrick (1) and Julie B. Staunton \\ Department of Physics, University of Warwick, Coventry CV4 7AL, United Kingdom
}

(Received 10 July 2019; published 3 October 2019)

\begin{abstract}
Computational design of more efficient rare earth/transition metal (RE-TM) permanent magnets requires accurately calculating the magnetocrystalline anisotropy (MCA) at finite temperature, since this property places an upper bound on the coercivity. Here, we present a first-principles methodology to calculate the MCA of RE-TM magnets which fully accounts for the effects of temperature on the underlying electrons. The itinerant electron TM magnetism is described within the disordered local moment picture, and the localized RE- $4 f$ magnetism is described within crystal field theory. We use our model, which is free of adjustable parameters, to calculate the MCA of the $R \mathrm{Co}_{5}(R=\mathrm{Y}$, La-Gd) magnet family for temperatures $0-600 \mathrm{~K}$. We correctly find a huge uniaxial anisotropy for $\mathrm{SmCo}_{5}\left(21.3 \mathrm{MJ} \mathrm{m}^{-3}\right.$ at $\left.300 \mathrm{~K}\right)$ and two finite temperature spin reorientation transitions for $\mathrm{NdCo}_{5}$. The calculations also demonstrate dramatic valency effects in $\mathrm{CeCo}_{5}$ and $\mathrm{PrCo}_{5}$. Our calculations provide quantitative, first-principles insight into several decades of RE-TM experimental studies.
\end{abstract}

DOI: 10.1103/PhysRevMaterials.3.101401

The excellent properties of rare earth/transition metal (RE-TM) permanent magnets have facilitated a number of technological revolutions in the last 50 years. Now, the urgent need for a low carbon, low emission economy is driving a global research effort dedicated to improving RE-TM performance for more efficient deployment in the drive motors of hybrid and electric vehicles [1]. RE-TM magnets combine the large volume magnetization and high Curie temperature of the elemental TM magnets Fe or Co with the potentially huge magnetocrystalline anisotropy (MCA) of the REs [2]. The REs consist of Sc, Y, and the lanthanides La-Lu, but it is $\mathrm{Y}$ and the light lanthanides, i.e., those with smaller atomic masses than $\mathrm{Gd}$, which are most attractive for applications due to their lower criticality [3]. Nd and Sm stand out thanks to the highly successful Nd-Fe-B and Sm-Co magnets [4-7], but Ce has the attraction of having a low cost and high abundance compared to the other REs [8].

Traditionally, RE-TM magnet research has been driven by experiments. First-principles computational modeling can uncover fundamental physical principles and provide new directions for RE-TM magnet design [9,10], but faces two challenges. First, RE-TM magnetism originates from both itinerant electrons and more localized lanthanide $4 f$ electrons [11]. Although the local spin-density approximation to density-functional theory [12] (DFT) satisfactorily describes the itinerant electrons, the $4 f$ electrons require specialist techniques like dynamical mean-field theory $[13,14]$, the local self-interaction correction (LSIC) [15], the open-core approximation [16-18], or Hubbard $+U$ models [19,20]. Second, DFT calculations are most easily performed at zero temperature, but under actual operating conditions the RE-TM magnetic moments are subject to a considerable level of thermal disorder [21]. The disordered local moment (DLM) picture

\footnotetext{
*c.patrick.1@warwick.ac.uk
}

accounts for this disorder within DFT [21] and, combined with the LSIC has been used successfully to calculate magnetizations, Curie temperatures, and phase diagrams of itinerant electron and RE-based magnets [22-24]. DFT-DLM studies of the MCA have also been performed on itinerant electron and Gd-based magnets [25-27], but it is important to realize that these materials are special cases, where there is no contribution to the MCA from $4 f$ electrons interacting with their local environment (the crystal field). A first-principles, finitetemperature theory which accounts for these crystal field effects (and is therefore applicable to general RE-TM magnets like $\mathrm{Nd}-\mathrm{Fe}-\mathrm{B}$ or $\mathrm{Sm}-\mathrm{Co}$ ) has, up to now, proven elusive.

In this Rapid Communication, we rectify this situation and demonstrate a first-principles theory of the MCA of RETM magnets including the crystal field interaction. Fundamentally, the theory takes a model originally developed by experimentalists, and obtains all of the quantities required by the model from DFT-based calculations. We demonstrate the theory on the $R \mathrm{Co}_{5}$ family of magnets, $(R=\mathrm{Y}, \mathrm{La}, \mathrm{Ce}, \mathrm{Pr}, \mathrm{Nd}$, $\mathrm{Sm}$, and $\mathrm{Gd}$ ). The $R \mathrm{Co}_{5}$ phase, shown in Fig. 1, is important due to its presence in $\mathrm{SmCo}_{5}$ and in the cell-boundary phase of commercial $\mathrm{Sm}_{2} \mathrm{Co}_{17}[7,28,29]$. We calculate anisotropy constants and spin reorientation transition temperatures to analyze experimental data obtained 40 years ago [30,31].

The model partitions the RE-TM magnet into an itinerant electron subsystem (originating from the TM, and RE valence electrons), and a subsystem of strongly localized RE- $4 f$ electrons [32]. Critically, the RE ions tend to adopt a 3+ state with a common $s^{2} d$ valence structure [33]. As a consequence, for each RE-TM magnet class the itinerant electron subsystem is essentially independent of the specific RE [32], so its properties can be obtained for the most computationally convenient prototype (e.g., $R=\mathrm{Y}$ or $\mathrm{Gd}$ ). The itinerant electrons drive the overall magnetic order, primarily determining the Curie temperature $T_{\mathrm{C}}$; for example, $T_{\mathrm{C}}$ differs by only $20 \mathrm{~K}$ in $\mathrm{Y}_{2} \mathrm{Fe}_{14} \mathrm{~B}$ and $\mathrm{Nd}_{2} \mathrm{Fe}_{14} \mathrm{~B}$ [34]. The itinerant electrons also drive the 

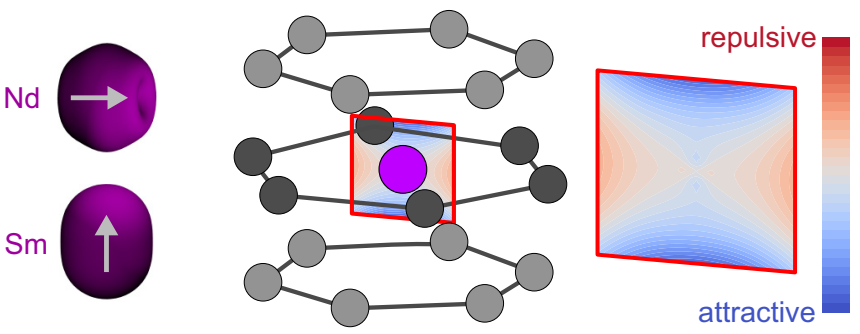

FIG. 1. Preferred orientation of RE- $4 f$ charge clouds (with magnetic moments indicated by arrows) for $\mathrm{Nd}$ and $\mathrm{Sm}$ in the $\mathrm{RCo}_{5}$ crystal field, shown on the contour plot. The Co and RE atoms are shaded in gray and purple, respectively. Repulsive (attractive) corresponds to high (low) potential energy.

RE-4 $f$ magnetic ordering through an antiferromagnetic exchange interaction [35], with an exchange field of a few hundred Tesla at cryogenic temperatures [36]. RE-RE interactions are relatively weak [37].

The RE-4 $f$ subsystem contributes to the magnetic moment and can have a small effect on $T_{\mathrm{C}}$ [24], but its most important contribution is to the MCA, which in turn provides an intrinsic mechanism for coercivity [38]. The origin of the potentially huge MCA of RE-TM magnets is illustrated in Fig. 1. The itinerant electrons and surrounding ions set up a (primarily) electrostatic potential with the symmetry of the RE site [32], known as the crystal field $(\mathrm{CF})$. The $\mathrm{CF}$ calculated for $\mathrm{RCO}_{5}$ is shown as a contour plot in Fig. 1, with electrons attracted to the blue regions and repelled by the red. Meanwhile the unfilled RE- $4 f$ shells form non-spherically-symmetric charge clouds coupled to the magnetic moment direction through a strong spin-orbit interaction [32]. The charge clouds are elongated either parallel or perpendicular to the moment direction depending on Hund's rules, with the opposing examples of $\mathrm{Sm}$ and $\mathrm{Nd}$ shown in Fig. 1. Placed in the $\mathrm{RCo}_{5} \mathrm{CF}$, the charge clouds will preferentially orientate with their elongated part lying in the attractive region, generating the MCA [39]. A secondary contribution to the MCA is provided by the itinerant electrons, with $\mathrm{YCo}_{5}$ (which has no RE- $4 f$ electrons) having a MCA energy of $5 \mathrm{MJ} \mathrm{m}^{-3}$ at room temperature [30], ten times larger than hexagonal Co [40].

The above ideas are formulated mathematically by introducing a Hamiltonian for the RE-4 $f$ electrons $\hat{\mathcal{H}}$ [41]:

$$
\hat{\mathcal{H}}=\lambda \hat{\mathbf{L}} \cdot \hat{\mathbf{S}}+\mu_{0} \mu_{B}(\hat{\mathbf{L}}+2 \hat{\mathbf{S}}) \cdot \mathbf{H}+2 \mu_{B} \hat{\mathbf{S}} \cdot \mathbf{B}_{\text {exch }}+\sum_{i} V\left(\mathbf{r}_{\mathbf{i}}\right) .
$$

Here, $\hat{\mathbf{L}}$ and $\hat{\mathbf{S}}$ are the orbital and spin angular momentum operators, where for each $R^{3+}$ ion $L$ and $S$ are fixed by Hund's rules. $\lambda$ quantifies the spin-orbit interaction, and $\mu_{B}$ is the Bohr magneton. $\mathbf{H}$ is an external magnetic field, and $\mathbf{B}_{\text {exch }}$ is the exchange field originating from the itinerant electrons. $V\left(\mathbf{r}_{i}\right)$ is the crystal field potential, where $i$ labels each $4 f$ electron. The Hamiltonian in Eq. (1) acts upon the RE-4 $f$ wave function which can be expressed schematically as a radial part multiplied by the angular part $\left|J, L, S, M_{J}\right\rangle$, where the quantities within the ket are standard quantum numbers [32]. Equation (1) is diagonalized within the manifold of states $\left|J, L, S, M_{J}=J, J-1, J-2, \ldots,-\right| J|\rangle$. We consider the ground $J=|L-S|$ multiplet, along with the first excited multiplet $J=|L-S|+1$ for Pr and $\mathrm{Nd}$, and also the next excited multiplet $J=|L-S|+2$ for Sm. Angular parts of the matrix elements of the CF term in Eq. (1) are calculated by decomposing the states into $\left|L, S, M_{L}, M_{S}\right\rangle$ form, and then using the operator form of the potential [42], which introduces Clebsch-Gordan coefficients and $l$-dependent (Stevens) prefactors $[32,43]$. The radial parts are incorporated into the $\mathrm{CF}$ coefficients $B_{l m}$ [33], described in more detail below. For a given temperature $T$, we use the eigenvalue spectrum of $\hat{\mathcal{H}}$ to construct the partition function $Z_{\mathrm{RE}}$ and the RE- $4 f$ free energy contribution $F_{\mathrm{RE}}\left(T, \mathbf{B}_{\text {exch }}, \mathbf{H}\right)=-k_{\mathrm{B}} T \ln Z_{\mathrm{RE}}$.

The quantities forming the itinerant contribution to the free energy $F_{\text {itin }}$ are determined from DFT-DLM calculations. $F_{\text {itin }}$ depends on the Co magnetization $\mathbf{M}_{\mathrm{Co}}$ :

$$
\begin{aligned}
& F_{\text {itin }}\left(T, \hat{\mathbf{M}}_{\mathrm{Co}}, \mathbf{H}\right) \\
& \quad=K_{1} \sin ^{2} \theta+K_{2} \sin ^{4} \theta-\mu_{0} M_{\mathrm{Co}}^{0}\left(1-p \sin ^{2} \theta\right) \hat{\mathbf{M}}_{\mathrm{Co}} \cdot \mathbf{H},
\end{aligned}
$$

where $K_{1}$ and $K_{2}$ quantify the itinerant electron contribution to the anisotropy, and $\cos \theta=\hat{\mathbf{M}}_{\mathrm{Co}} \cdot \hat{\mathbf{z}}$, with $\hat{\mathbf{z}}$ pointing along the $c$ axis. $p$ quantifies the magnetization anisotropy, which in the $\mathrm{RCO}_{5}$ compounds can cause the Co magnetization to reduce by a few percent from its maximum value $M_{\text {Co }}^{0}$ [44]. $F_{\text {itin }}$ depends on temperature through the quantities $K_{1}, K_{2}$, $M_{\mathrm{Co}}^{0}$, and $p$.

The two subsystems are coupled together by noting that $\hat{\mathbf{M}}_{\mathrm{Co}}=-\hat{\mathbf{B}}_{\text {exch }}$, i.e., the exchange field felt by the RE- $4 f$ electrons points antiparallel to the Co magnetization [16]. The equilibrium direction of $\mathbf{M}_{\mathrm{Co}}$ (equivalently, of $\mathbf{B}_{\text {exch }}$ ) minimizes the sum of $F_{\text {itin }}$ and $F_{\mathrm{RE}}$. The RE magnetization is obtained as $\mathbf{M}_{\mathrm{RE}}=-\mu_{B}\langle\hat{\mathbf{L}}+2 \hat{\mathbf{S}}\rangle_{T}$, with $\langle\cdot\rangle_{T}$ denoting the thermal average over the eigenvalue spectrum of Eq. (1) at the equilibrium value of $\mathbf{B}_{\text {exch }}$, and the total magnetization is $\mathbf{M}_{\mathrm{Tot}}=\mathbf{M}_{\mathrm{RE}}+\mathbf{M}_{\mathrm{Co}}$. The magnetization measured along the field direction $M$ is $\mathbf{M}_{\mathrm{Tot}} \cdot \hat{\mathbf{H}}$, whilst the easy direction of magnetization $\alpha$ is obtained as $\cos ^{-1}\left(\hat{\mathbf{M}}_{\mathrm{Tot}} \cdot \hat{\mathbf{z}}\right)$ in zero external field.

$\mathbf{B}_{\text {exch }}, K_{1}, K_{2}, M_{\mathrm{Co}}^{0}$, and $p$ are obtained using the FPMVB procedure developed to calculate first-principles magnetization vs $B$-field curves for $\mathrm{GdCo}_{5}$ [27]. FPMVB fits DFT-DLM torque calculations [45] for different magnetic configurations to extract the desired quantities $[27,46]$. For all $R^{3+} \mathrm{Co}_{5}$ compounds considered here, we exploit the isovalence of the $R^{3+}$ ions and substitute the RE with Gd in the FPMVB calculations. This step ensures no erroneous double counting of the CF contribution which is already accounted for by $F_{\mathrm{RE}}$, but still captures the coupling between TM-3d and RE-5d valence states. We do, however, use the (experimental) lattice parameters appropriate for each RE $[47,48] . K_{1}, K_{2}, M_{\mathrm{Co}}^{0}$, and $p$ were fitted to calculations where $\theta$ was varied between 0 and $90^{\circ}$ in $10^{\circ}$ intervals. $\mathbf{B}_{\text {exch }}$ was obtained by fitting the torque induced by introducing a $1^{\circ}$ canting between the $\mathrm{Gd}$ and Co sublattices to a free energy of the form $-\mathbf{B}_{\text {exch }} \cdot \mathbf{M}_{\mathrm{Gd}, \mathrm{s}}$ where $\mathbf{M}_{\mathrm{Gd}, \mathrm{s}}$ is the spin moment of $\mathrm{Gd}$ including thermal disorder, i.e., the local spin moment weighted by the $\mathrm{Gd}$ order parameter [46]. For itinerant $\mathrm{CeCo}_{5}$ and $\mathrm{PrCo}_{5}$ (see below), the quantities in Eq. (2) were obtained directly from DFTDLM calculations on these compounds. The calculations used 
the atomic sphere approximation, angular momentum expansions with maximum $l=3$, and an adaptive reciprocal space sampling to ensure high precision [49]. Exchange and correlation were modeled within the local spin-density approximation (LSDA) [50], with an orbital polarization correction applied to the Co- $d$ electrons [51] and the LSIC applied to Gd. The calculated quantities are given as Supplemental Material [52].

We calculate the $R \mathrm{Co}_{5} \mathrm{CF}$ coefficients using an yttriumanalog model [33]. The basic premise here is that due to the isovalence of $R^{3+}$ ions, the $R^{3+} \mathrm{Co}_{5} \mathrm{CF}$ potential (which originates from the valence electronic structure) can be substituted with that of $\mathrm{Y}^{3+} \mathrm{Co}_{5}$. This step ensures no double counting of RE- $4 f$ electrons, and allows the use of projector-augmented wave-based DFT calculations to calculate the CF potential to high accuracy without needing special methods to treat the $4 f$ electrons [33]. The CF potential is combined with the radial RE- $4 f$ wave functions obtained in LSIC calculations. At the RE site (symmetry $D_{6 h}$ ) there are four independent components of the CF potential which affect the $4 f$ anisotropy, with $(l, m)=(2,0),(4,0),(6,0)$, and $(6,6)[=(6,-6)]$. The calculated CF coefficients are given as Supplemental Material [52]. We note that this method implicitly neglects any temperature dependence of the $\mathrm{CF}$ coefficients themselves, and future work must evaluate the effects of finite temperature, e.g., due to charge fluctuations or lattice expansion [47]. The calculations were performed using the GPAW code [53] within the LSDA. A plane wave basis set with a kinetic energy cutoff of $1200 \mathrm{eV}$ was used, and reciprocal space sampling performed on a $20 \times 20 \times 20$ grid. The spin-orbit parameter $\lambda$ was calculated using the RE-centered spherical potential $V_{0}(r)$ from the LSIC calculation as $\lambda=\int d r r^{2} n_{4 f}^{0}(r) \zeta(r) /(2 S)$ [54], where the normalized spherically symmetric $4 f$ density $n_{4 f}^{0}(r)$ was also obtained from the LSIC calculation [33] and $\zeta(r)=\frac{\hbar^{2}}{2 m^{2} c^{2}} \frac{1}{r} \frac{\partial V_{0}}{\partial r}$. The calculated values of $\lambda$ are 1205, 703, 540 , and $417 \mathrm{~K}$ for $\mathrm{Ce}, \mathrm{Pr}, \mathrm{Nd}$, and $\mathrm{Sm}$, respectively. These $\lambda$ values yield anisotropy constants indistinguishable from those calculated using experimental $\lambda$ values extracted from spectroscopic measurements $[41,54]$.

We now demonstrate the theory by calculating experimentally measurable quantities. Figure 2 (left panel) reproduces anisotropy constants measured by Ermolenko in 1976 [30] for $\mathrm{YCo}_{5}, \mathrm{LaCo}_{5}, \mathrm{NdCo}_{5}, \mathrm{SmCo}_{5}$, and $\mathrm{GdCo}_{5}$. They were extracted using the Sucksmith-Thompson (ST) method [40], which is based on the expression for the dependence of the free energy of a uniaxial ferromagnet on the magnetization direction $\Theta$ :

$$
F_{\mathrm{FM}}(\Theta)=\kappa_{1} \sin ^{2} \Theta+\kappa_{2} \sin ^{4} \Theta+\mathcal{O}\left(\sin ^{6} \Theta\right) .
$$

As explained in detail in the Supplemental Material [52], measuring the magnetization along the hard direction and plotting the data as an Arrott plot $\left(H / M\right.$ vs $\left.M^{2}\right)$ [55] allows $\kappa_{1}$ and $\kappa_{2}$ to be extracted from the gradient and intercept. Equation (3) and the ST method strictly apply to ferromagnets, but the same technical procedure can be applied to RE-TM ferrimagnets too [27]. However, the fact that the external field can induce a canting between the RE and TM moments means that the extracted anisotropy constants for the ferrimagnet are effective ones, which measure both the anisotropy of

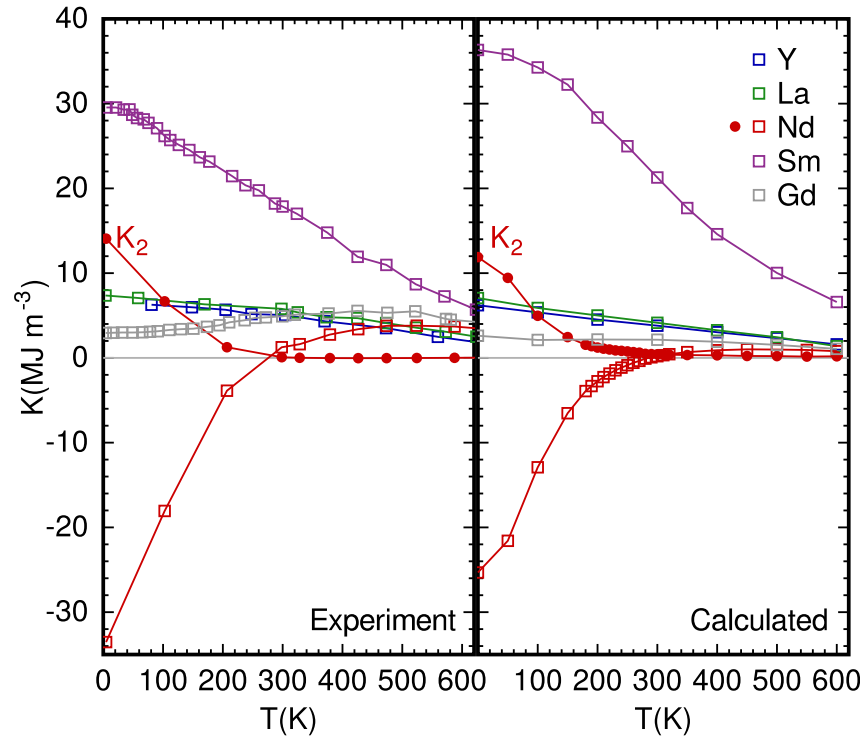

FIG. 2. Experimental anisotropy constants $\kappa_{1}$ (and $\kappa_{2}$ for $\mathrm{NdCo}_{5}$ ) [30] of $\mathrm{RCo}_{5}$, compared to the current calculations.

the individual sublattices and the strength of the exchange interaction keeping the spin moments antialigned $[27,46]$.

The experimental data in Fig. 2 demonstrates the diversity in $\kappa$ among $R \mathrm{Co}_{5}$. The behavior of $\mathrm{YCo}_{5}$ and $\mathrm{LaCo}_{5}$, where the $\mathrm{RE}$ is nonmagnetic, is rather similar. Both compounds display uniaxial anisotropy associated with the itinerant electron subsystem. $\mathrm{GdCo}_{5}$ is still uniaxial, but is softer than $\mathrm{YCo}_{5}$ and $\mathrm{LaCo}_{5}$. Since the filled $\mathrm{Gd}-4 f$ subshell makes no CF contribution to the anisotropy, this reduction in $\kappa_{1}$ is due to the field-induced canting of the Gd and Co magnetic moments [27]. $\mathrm{SmCo}_{5}$ stands out for having the largest uniaxial anisotropy over the entire temperature range, with a room temperature value of $17.9 \mathrm{MJ} \mathrm{m}^{-3}$ [30]. $\mathrm{NdCo}_{5}$ has a negative $\kappa_{1}$ at low temperatures which switches to positive at approximately $280 \mathrm{~K}$, and also has a non-negligible $\kappa_{2}$, at variance with the other compounds. As discussed below, this variation results in $\mathrm{NdCo}_{5}$ undergoing spin reorientation transitions from planar $\rightarrow$ cone $\rightarrow$ uniaxial anisotropy [31].

The right panel of Fig. 2 is the main result of this work, showing the anisotropy constants obtained entirely from first principles. We calculated hard-axis magnetization curves, and then performed the ST analysis on the data to extract $\kappa_{1}$ and $\kappa_{2}$, in exact correspondence with the experimental procedure [52]. The calculations reproduce all of the experimentally observed behavior. $\mathrm{SmCo}_{5}$ and $\mathrm{NdCo}_{5}$ have strong uniaxial and planar anisotropy at zero temperature, respectively. $\mathrm{NdCo}_{5}$ has a non-negligible $\kappa_{2}$ and a value of $\kappa_{1}$ which changes sign between 280 and $290 \mathrm{~K}$. $\mathrm{YCo}_{5}$ and $\mathrm{LaCo}_{5}$ have uniaxial anisotropy, with $\mathrm{LaCo}_{5}$ slightly stronger. $\mathrm{GdCo}_{5}$ also has uniaxial anisotropy but is softer, and has a complicated temperature dependence.

Comparing in more detail, we find agreement between experimental and calculated $\kappa$ values to within a few $\mathrm{MJ} \mathrm{m}^{-3}$ for all but the lowest temperatures, where the classical statistical mechanics of DFT-DLM calculations leads to inaccuracies [37], and high temperatures, where experimentally the compounds might undergo decomposition [27]. We calculate 


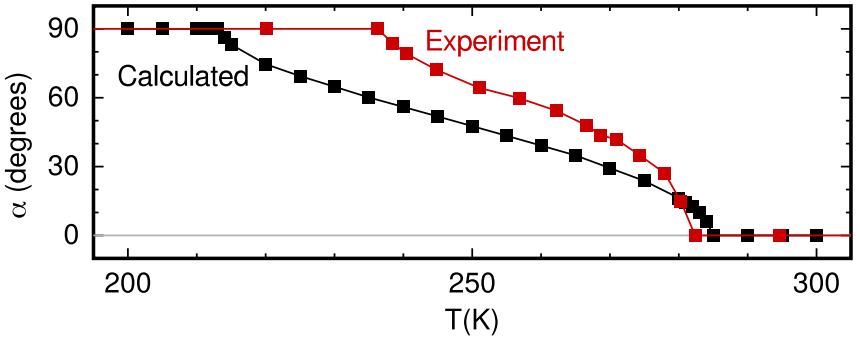

FIG. 3. Evolution of the easy magnetization direction $\alpha$ in $\mathrm{NdCo}_{5}$, as reported experimentally [31] and calculated here.

$\kappa_{1}$ at room temperature for $\mathrm{SmCo}_{5}$ to be $21.3 \mathrm{MJ} \mathrm{m}^{-3}$ (experiment $17.9 \mathrm{MJ} \mathrm{m}^{-3}$ ). Even at zero temperature the value of $36.3 \mathrm{MJ} \mathrm{m}^{-3}$ shows improved agreement with experiment (29.5 $\left.\mathrm{MJ} \mathrm{m}^{-3}\right)$ compared to open core $\left(19.7 \mathrm{MJ} \mathrm{m}^{-3}\right.$ ) [17] or Hubbard $+U$ calculations $\left(40.5 \mathrm{MJ} \mathrm{m}^{-3}\right)$ [20].

The calculations also reproduce more subtle features, for instance the slightly enhanced anisotropy (by less than $1 \mathrm{MJ} \mathrm{m}^{-3}$ ) of $\mathrm{LaCo}_{5}$ over $\mathrm{YCo}_{5}$. The least good agreement is for $\mathrm{GdCo}_{5}$, especially at higher temperatures; however, more recent measurements of $\kappa_{1}$ found different behavior at elevated temperatures [27,30]. At lower temperatures, we note that the present calculations do not include the magnetostatic dipole-dipole contribution to the MCA, or the Gd-5d contribution to the itinerant electron anisotropy, which we previously calculated to be $24 \%$ the size of the Co contribution [27]. We conclude that omitting the magnetostatic and RE- $d$ contribution to the anisotropy is reasonable for nonmagnetic REs or those with unfilled $4 f$ shells (whose RE- $4 f$ anisotropy is much larger), but is less suitable for Gd-based magnets.

Unlike the other materials in Fig. 2, the easy direction of magnetization of $\mathrm{NdCo}_{5}$ lies in the $a b$ plane at low temperature, with polar angle $\alpha=90^{\circ}$. The anisotropy within the $a b$ plane is determined by the $B_{6 \pm 6} \mathrm{CF}$ coefficients. Both our calculations and experiments find the easy direction to be the $a$ axis, which points from the RE to between its nearestneighbor Co atoms [56]. Experimentally, as $T$ is increased from zero to past approximately $240 \mathrm{~K}$, a spin reorientation transition (SRT) occurs and the magnetization begins to rotate towards the $c$ axis, i.e., planar $\rightarrow$ cone anisotropy. This rotation continues (decreasing $\alpha$ ) until approximately $280 \mathrm{~K}$, when a second SRT (cone $\rightarrow$ uniaxial) occurs. Further increasing $T$ sees $\alpha$ remaining at $0^{\circ}$ up to $T_{\mathrm{C}}$. The presence of the SRTs close to room temperature led to the proposal that $\mathrm{NdCo}_{5}$ may be a candidate material for magnetic refrigeration [57]. The evolution of $\alpha$ as measured experimentally in Ref. [31] is shown in Fig. 3.

The calculated variation of $\alpha$ with temperature is also shown in Fig. 3. We see that the agreement with experiment is remarkably good, with calculated SRT temperatures of $T_{\mathrm{SRT} 1}=214 \mathrm{~K}$ (plane $\rightarrow$ cone) and $T_{\mathrm{SRT} 2}=285 \mathrm{~K}$ (cone $\rightarrow$ uniaxial). There is also good agreement between calculated and experimental $\kappa$ values, especially in the SRT region. Indeed, the SRTs are intimately linked to the temperature dependence of $\kappa_{1}$ and $\kappa_{2}$, with the plane-cone SRT occurring when $\kappa_{1}=-2 \kappa_{2}$ and the cone-axis SRT occurring when $\kappa_{1}$ crosses zero [58,59].

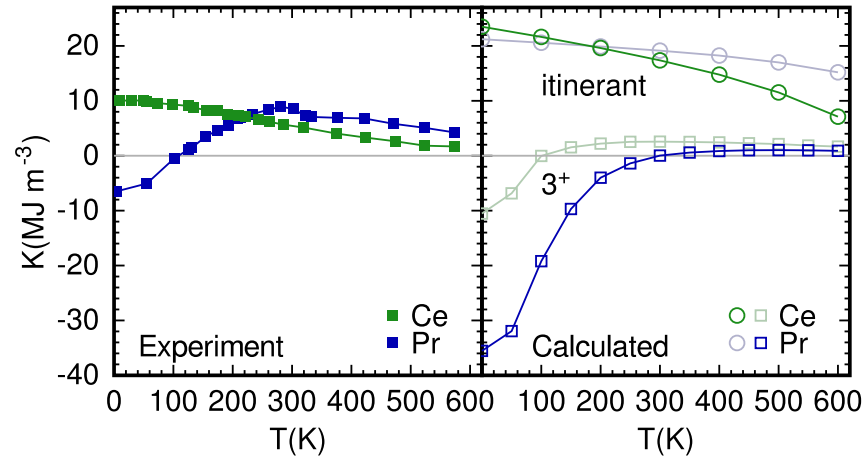

FIG. 4. Comparison of experimental and calculated anisotropy constants for $\mathrm{CeCo}_{5}$ and $\mathrm{PrCo}_{5}$. Values are shown for both itinerant and localized (RE in $3+$ state) $4 f$ electrons, with the ground state predicted by the LSIC calculations shown in the darker color.

The calculations provide the underlying physical explanation of the SRTs, which result from a competition between the uniaxial anisotropy of the itinerant electrons and a preference for the oblate $\mathrm{Nd}^{3+}$ charge cloud to have its axis lying in the $a b$ plane (Fig. 1). As the temperature increases, the Nd moments disorder more quickly than the $\mathrm{Co}$, due to the relative weakness of the RE-TM exchange field $\mathbf{B}_{\text {exch }}$ compared to the exchange interaction between itinerant moments [24]. As a result, the negative contribution to $\kappa_{1}$ from $\mathrm{Nd}$ reduces quickly with temperature, leaving the positive uniaxial contribution from the itinerant electrons. Obtaining realistic SRT temperatures therefore requires accounting for the itinerant electron anisotropy, the crystal field potential, and the exchange field at a comparable level of accuracy.

We finally consider $\mathrm{CeCO}_{5}$ and $\mathrm{PrCO}_{5}$. Ce has a wellknown tendency to undergo transitions between trivalent and tetravalent valence states, as seen for instance in the $\alpha-\gamma$ transition [60,61]. The LSIC describes strongly correlated RE-4 $f$ electrons, with them forming a narrow band several $\mathrm{eV}$ below the Fermi level [24]. Without the LSIC, the $4 f$ electrons are less correlated and more itinerant, appearing as wider bands close to the Fermi level. The LSIC finds a lower-energy ground state if the enhanced correlation offsets the energy penalty associated with the stronger localization $[15,24]$. Of the $R \mathrm{Co}_{5}$ compounds, the LSIC predicts a higherenergy ground state only for $\mathrm{CeCo}_{5}$, implying that the $\mathrm{Ce}-4 f$ electron is not strongly localized.

Practically, we model compounds with more itinerant (weakly correlated) RE-4 $f$ electrons by performing non-LSIC DFT-DLM calculations on $\mathrm{RCo}_{5}$, with only $F_{\text {itin }}$ contributing to the free energy. The values of $\kappa_{1}$ calculated in this way for $\mathrm{CeCO}_{5}$ and $\mathrm{PrCO}_{5}$ are labeled "itinerant" in Fig. 4. We also show $\kappa_{1}$ values labeled " 3 ", , calculated for strongly localized RE-4 $f$ electrons using the same method as in Fig. 2. The Ce and Pr moments are held collinear to the Co moments in the itinerant calculations [52].

Figure 4 shows a dramatic difference in the anisotropy constants calculated for the different RE- $4 f$ valences. $\mathrm{Pr}^{3+} \mathrm{Co}_{5}$ has an $a b$ plane anisotropy at low temperature, which is stronger than $\mathrm{NdCo}_{5}$. This behavior is in fact expected; the leading crystal field contribution to the MCA scales as $J\left(J-\frac{1}{2}\right) \alpha_{J}\left(\alpha_{J}\right.$ being the Stevens factor), and this quantity 
is larger for Pr than Nd [32]. The calculated plane $\rightarrow$ cone and cone $\rightarrow$ uniaxial SRTs occur at 235 and $297 \mathrm{~K}$, respectively, which are higher temperatures than those calculated for $\mathrm{NdCo}_{5} . \mathrm{Ce}^{3+} \mathrm{Co}_{5}$ meanwhile is calculated to have cone anisotropy at zero temperature, with $\alpha=80^{\circ}$. A cone $\rightarrow$ axis SRT occurs at $100 \mathrm{~K}$, after which the compound has uniaxial anisotropy. The presence of only one SRT shows that $\mathrm{Ce}^{3+}$ has a weaker planar anisotropy than $\mathrm{Pr}^{3+}$ or $\mathrm{Nd}^{3+}$. This weaker anisotropy is due to $\mathrm{CeCo}_{5}$ having a reduced $B_{20}$ CF coefficient, which correlates with a contracted $a$ lattice parameter [52].

If instead the RE- $4 f$ electrons are treated as itinerant, both $\mathrm{CeCo}_{5}$ and $\mathrm{PrCo}_{5}$ are found to have strong uniaxial anisotropy. At low temperatures $\mathrm{CeCo}_{5}$ has the higher value of $\kappa_{1}$ $\left(23.5 \mathrm{MJ} \mathrm{m}^{-3}\right.$ at $0 \mathrm{~K}$ ), while above $200 \mathrm{~K}, \kappa_{1}$ of $\mathrm{PrCo}_{5}$ is larger. The $\kappa_{1}$ values exceed those calculated for $\mathrm{YCo}_{5}$ and $\mathrm{LaCo}_{5}$, showing that delocalizing the RE- $4 f$ electrons boosts the uniaxial anisotropy.

The experimental anisotropy constants from Ref. [30] are also shown in Fig. 4. The experiments support the picture obtained from the total energy calculations, that the Ce- $4 f$ and $\operatorname{Pr}-4 f$ electrons are more itinerant/localized (weakly/strongly correlated), respectively. $\mathrm{CeCo}_{5}$ has uniaxial anisotropy across the entire temperature range. For $\mathrm{PrCO}_{5}$, although $\kappa_{1}$ is negative at low temperature, its magnitude is smaller than that measured for $\mathrm{NdCo}_{5}\left(-6.5 \mathrm{MJ} \mathrm{m}^{-3}\right.$ vs $-33.5 \mathrm{MJ} \mathrm{m}^{-3}$ at $4 \mathrm{~K}$ ). As a result, at low temperature the easy magnetization direction of $\mathrm{PrCo}_{5}$ does not lie in the $a b$ plane, but rather in a cone with $\alpha=23^{\circ}$ [62]. Increasing the temperature decreases $\alpha$, and a cone-axis SRT occurs at $105 \mathrm{~K}$ [62]. Therefore, although the Pr ions do favor $a b$-plane anisotropy, their contribution is weaker than from the $\mathrm{Nd}$ ions in $\mathrm{NdCo}_{5}$, at variance with the $\mathrm{CF}$ picture. Overall, in $\mathrm{CeC}_{5}\left(\mathrm{PrCo}_{5}\right)$ experiments find a smaller uniaxial (planar) contribution from $\mathrm{Ce}\left(\mathrm{Pr}^{3+}\right)$. As a result, the calculated uniaxial anisotropy of $\mathrm{CeC}_{5}$ is larger than found experimentally, while the plane $\rightarrow$ cone SRT of $\mathrm{PrCo}_{5}$ at $235 \mathrm{~K}$ is missing from experiments.

The anomalous behavior of $\mathrm{PrCo}_{5}$ in the context of CF theory was identified in Ref. [63], where it was proposed that in $\mathrm{PrCo}_{5}$, $\mathrm{Pr}$ assumes a mixed valence state, e.g., $\mathrm{Pr}^{3.5+}$, whose properties lie between Pr and Ce. The calculations shown in Fig. 4 support this view, if we make the reasonable assumption that the anisotropic properties of the mixed valence state are bounded by those of the strongly localized and more itinerant (strongly and weakly correlated) $\operatorname{Pr}-4 f$ electrons. In a similar way, the experimentally observed reduction in $\mathrm{CeCo}_{5}$ uniaxial anisotropy compared to the calculations could be explained if the Ce- $4 f$ electron was more localized (correlated) than predicted by the "itinerant" calculations. From Fig. 4, such an electron would be expected to have a reduced contribution to the uniaxial anisotropy. Within this picture, encouraging the itineracy of the Ce- $4 f$ electron through, e.g., chemical pressure, would boost the uniaxial anisotropy of $\mathrm{CeCo}_{5}$.

Apart from highlighting the $4 f$-electron physics of Ce and $\mathrm{Pr}$, our calculations serve as a reminder of the remarkable properties of $\mathrm{SmCo}_{5}$. As well as its huge zero-temperature uniaxial anisotropy, the large spin moment of Sm strengthens its coupling to the exchange field, so that the Sm moments stay ordered up to higher temperatures. Furthermore, mixing of the higher- $J$ multiplets also boosts the anisotropy [64]. As a result, as shown in Fig. 2, the $\kappa_{1}$ value of $\mathrm{SmCo}_{5}$ remains larger than that of $\mathrm{YCo}_{5}$ and $\mathrm{LaCo}_{5}$ (where the RE is nonmagnetic), even at $600 \mathrm{~K}$. Previously we have shown that the electronic structure of $\mathrm{SmCo}_{5}$ close to the Fermi level also gives it the highest $T_{\mathrm{C}}$ of the $R \mathrm{Co}_{5}$ compounds [24].

In summary, we have demonstrated a framework to calculate the finite-temperature MCA of RE-TM magnets. Combined with the previously established DFT-DLM method which provides finite-temperature magnetization and $T_{\mathrm{C}}$ [24], we have a full framework to calculate the intrinsic properties of RE-TM magnets which requires no experimental input beyond the crystal structure. The validation of our method on the $\mathrm{RCO}_{5}$ magnet class opens the door to tackling other RE-TM magnets, such as Nd-Fe-B, $R \mathrm{Fe}_{12}$, and $\mathrm{Sm}_{2} \mathrm{Co}_{17}$. The good performance of the calculations for $\mathrm{SmCo}_{5}$ will allow us to propose strategies to improve this magnet, e.g., through modification of the CF potential and/or exchange field through TM doping or application of pressure. More generally, our work realizes the proposal made two decades ago in Ref. [65], which suggested that rather than trying to compare first-principles CF coefficients to experiment (themselves obtained by fitting), the comparison should instead be made for anisotropy constants.

The present work forms part of the PRETAMAG project, funded by the UK Engineering and Physical Sciences Research Council, Grant No. EP/M028941/1. We thank H. Akai and M. Matsumoto for useful discussions.
[1] H. Nakamura, The current and future status of rare earth permanent magnets, Scr. Mater. 154, 273 (2018).

[2] J. M. D. Coey, Hard magnetic materials: A perspective, IEEE Trans. Magn. 47, 4671 (2011).

[3] O. Gutfleisch, M. A. Willard, E. Brück, C. H. Chen, S. G. Sankar, and J. P. Liu, Magnetic materials and devices for the 21st century: Stronger, lighter, and more energy efficient, Adv. Mater. 23, 821 (2011).

[4] M. Sagawa, S. Fujimura, N. Togawa, H. Yamamoto, and Y. Matsuura, New material for permanent magnets on a base of Nd and Fe (invited), J. Appl. Phys. 55, 2083 (1984).
[5] J. J. Croat, J. F. Herbst, R. W. Lee, and F. E. Pinkerton, Pr-Fe and Nd-Fe-based materials: A new class of high-performance permanent magnets (invited), J. Appl. Phys. 55, 2078 (1984).

[6] K. Strnat, The hard-magnetic properties of rare earth-transition metal alloys, IEEE Trans. Magn. 8, 511 (1972).

[7] K. Strnat, G. Hoffer, J. Olson, W. Ostertag, and J. J. Becker, A family of new cobalt-base permanent magnet materials, J. Appl. Phys. 38, 1001 (1967).

[8] A. K. Pathak, M. Khan, K. A. Gschneidner, Jr., R. W. McCallum, L. Zhou, K. Sun, K. W. Dennis, C. Zhou, F. E. Pinkerton, M. J. Kramer, and V. K. Pecharsky, Cerium: An 
unlikely replacement of dysprosium in high performance NdFe-B permanent magnets, Adv. Mater. 27, 2663 (2015).

[9] O. Y. Vekilova, B. Fayyazi, K. P. Skokov, O. Gutfleisch, C. Echevarria-Bonet, J. M. Barandiarán, A. Kovacs, J. Fischbacher, T. Schrefl, O. Eriksson, and H. C. Herper, Tuning the magnetocrystalline anisotropy of $\mathrm{Fe}_{3} \mathrm{Sn}$ by alloying, Phys. Rev. B 99, 024421 (2019).

[10] Y. Tatetsu, Y. Harashima, T. Miyake, and Y. Gohda, Role of typical elements in $\mathrm{Nd}_{2} \mathrm{Fe}_{14} X(X=\mathrm{B}, \mathrm{C}, \mathrm{N}, \mathrm{O}, \mathrm{F})$, Phys. Rev. Mater. 2, 074410 (2018).

[11] J. P. Perdew and A. Zunger, Self-interaction correction to density-functional approximations for many-electron systems, Phys. Rev. B 23, 5048 (1981).

[12] W. Kohn and L. J. Sham, Self-consistent equations including exchange and correlation effects, Phys. Rev. 140, A1133 (1965).

[13] I. L. M. Locht, Y. O. Kvashnin, D. C. M. Rodrigues, M. Pereiro, A. Bergman, L. Bergqvist, A. I. Lichtenstein, M. I. Katsnelson, A. Delin, A. B. Klautau, B. Johansson, I. Di Marco, and O. Eriksson, Standard model of the rare earths analyzed from the Hubbard I approximation, Phys. Rev. B 94, 085137 (2016).

[14] P. Delange, S. Biermann, T. Miyake, and L. Pourovskii, Crystalfield splittings in rare-earth-based hard magnets: An ab initio approach, Phys. Rev. B 96, 155132 (2017).

[15] M. Lüders, A. Ernst, M. Däne, Z. Szotek, A. Svane, D. Ködderitzsch, W. Hergert, B. L. Györffy, and W. M. Temmerman, Self-interaction correction in multiple scattering theory, Phys. Rev. B 71, 205109 (2005).

[16] M. S. S. Brooks, L. Nordstrom, and B. Johansson, $3 d-5 d$ band magnetism in rare earth-transition metal intermetallics: Total and partial magnetic moments of the $R \mathrm{Fe}_{2}(R=\mathrm{Gd}-\mathrm{Yb})$ Laves phase compounds, J. Phys.: Condens. Matter 3, 2357 (1991).

[17] P. Söderlind, A. Landa, I. L. M. Locht, D. Åberg, Y. Kvashnin, M. Pereiro, M. Däne, P. E. A. Turchi, V. P. Antropov, and O. Eriksson, Prediction of the new efficient permanent magnet $\mathrm{SmCoNiFe}_{3}$, Phys. Rev. B 96, 100404(R) (2017).

[18] T. Fukazawa, H. Akai, Y. Harashima, and T. Miyake, Firstprinciples study of intersite magnetic couplings in $\mathrm{NdFe}_{12}$ and $\mathrm{NdFe}_{12} X(X=\mathrm{B}, \mathrm{C}, \mathrm{N}, \mathrm{O}, \mathrm{F})$, J. Appl. Phys. 122, 053901 (2017).

[19] O. Waller, P. Piekarz, A. Bosak, P. T. Jochym, S. Ibrahimkutty, A. Seiler, M. Krisch, T. Baumbach, K. Parlinski, and S. Stankov, Lattice dynamics of neodymium: Influence of $4 f$ electron correlations, Phys. Rev. B 94, 014303 (2016).

[20] P. Larson, I. I. Mazin, and D. A. Papaconstantopoulos, Effects of doping on the magnetic anisotropy energy in $\mathrm{SmCo}_{5-x} \mathrm{Fe}_{x}$ and $\mathrm{YCo}_{5-x} \mathrm{Fe}_{x}$, Phys. Rev. B 69, 134408 (2004).

[21] B. L. Györffy, A. J. Pindor, J. Staunton, G. M. Stocks, and H. Winter, A first-principles theory of ferromagnetic phase transitions in metals, J. Phys. F: Met. Phys. 15, 1337 (1985).

[22] I. D. Hughes, M. Daane, A. Ernst, W. Hergert, M. Lüders, J. Poulter, J. B. Staunton, A. Svane, Z. Szotek, and W. M. Temmerman, Lanthanide contraction and magnetism in the heavy rare earth elements, Nature (London) 446, 650 (2007).

[23] E. Mendive-Tapia and J. B. Staunton, Theory of Magnetic Ordering in the Heavy Rare Earths: Ab Initio Electronic Origin of Pair- and Four-Spin Interactions, Phys. Rev. Lett. 118, 197202 (2017).

[24] C. E. Patrick and J. B. Staunton, Rare-earth/transitionmetal magnets at finite temperature: Self-interaction-corrected relativistic density functional theory in the disordered local moment picture, Phys. Rev. B 97, 224415 (2018).

[25] J. B. Staunton, S. Ostanin, S. S. A. Razee, B. L. Györffy, L. Szunyogh, B. Ginatempo, and E. Bruno, Temperature Dependent Magnetic Anisotropy in Metallic Magnets from an $A b$ Initio Electronic Structure Theory: $L 1_{0}$-Ordered FePt, Phys. Rev. Lett. 93, 257204 (2004).

[26] M. Matsumoto, R. Banerjee, and J. B. Staunton, Improvement of magnetic hardness at finite temperatures: Ab initio disordered local-moment approach for $\mathrm{YCo}_{5}$, Phys. Rev. B 90, 054421 (2014).

[27] C. E. Patrick, S. Kumar, G. Balakrishnan, R. S. Edwards, M. R. Lees, L. Petit, and J. B. Staunton, Calculating the Magnetic Anisotropy of Rare-Earth-Transition-Metal Ferrimagnets, Phys. Rev. Lett. 120, 097202 (2018).

[28] J. H. Wernick and S. Geller, Transition element-rare earth compounds with $\mathrm{Cu}_{5} \mathrm{Ca}$ structure, Acta Cryst. 12, 662 (1959).

[29] K. Kumar, RETM 5 and $\mathrm{RE}_{2} \mathrm{TM}_{17}$ permanent magnets development, J. Appl. Phys. 63, R13 (1988).

[30] A. Ermolenko, Magnetocrystalline anisotropy of rare earth intermetallics, IEEE Trans. Magn. 12, 992 (1976).

[31] H. P. Klein, A. Menth, and R. S. Perkins, Magnetocrystalline anisotropy of light rare-earth cobalt compounds, Physica B+C 80, 153 (1975).

[32] M. D. Kuz'min and A. M. Tishin, Theory of crystal field effects in 3d-4f intermetallic compounds, in Handbook of Magnetic Materials, edited by K. H. J. Buschow (Elsevier, Amsterdam, 2008), Vol. 17, Chap. 3, p. 149.

[33] C. E. Patrick and J. B. Staunton, Crystal field coefficients for yttrium analogues of rare-earth/transition-metal magnets using density-functional theory in the projector-augmented wave formalism, J. Phys.: Condens. Matter 31, 305901 (2019).

[34] J. F. Herbst, $\mathrm{R}_{2} \mathrm{Fe}_{14} \mathrm{~B}$ materials: Intrinsic properties and technological aspects, Rev. Mod. Phys. 63, 819 (1991).

[35] M. S. S. Brooks, O. Eriksson, and B. Johansson, 3d-5d band magnetism in rare earth transition metal intermetallics: $\mathrm{LuFe}_{2}$, J. Phys.: Condens. Matter 1, 5861 (1989).

[36] M. Loewenhaupt, P. Tils, K. Buschow, and R. Eccleston, Intersublattice exchange coupling in Gd-Co compounds studied by INS, J. Magn. Magn. Mater. 138, 52 (1994).

[37] C. E. Patrick, S. Kumar, G. Balakrishnan, R. S. Edwards, M. R. Lees, E. Mendive-Tapia, L. Petit, and J. B. Staunton, Rare-earth/transition-metal magnetic interactions in pristine and $(\mathrm{Ni}, \mathrm{Fe})$-doped $\mathrm{YCo}_{5}$ and $\mathrm{GdCo}_{5}$, Phys. Rev. Mater. 1, 024411 (2017).

[38] W. F. Brown, Criterion for uniform micromagnetization, Phys. Rev. 105, 1479 (1957).

[39] D. J. Newman and B. Ng, The superposition model of crystal fields, Rep. Prog. Phys. 52, 699 (1989).

[40] W. Sucksmith and J. E. Thompson, The magnetic anisotropy of cobalt, Proc. R. Soc. A 225, 362 (1954).

[41] Z. Tie-song, J. Han-min, G. Guang-hua, H. Xiu-feng, and C. Hong, Magnetic properties of $R$ ions in $R \mathrm{Co}_{5}$ compounds ( $R=$ Pr, Nd, Sm, Gd, Tb, Dy, Ho, and Er), Phys. Rev. B 43, 8593 (1991).

[42] K. W. H. Stevens, Matrix elements and operator equivalents connected with the magnetic properties of rare earth ions, Proc. Phys. Soc. A 65, 209 (1952). 
[43] N. F. Chilton, R. P. Anderson, L. D. Turner, A. Soncini, and K. S. Murray, PHI: A powerful new program for the analysis of anisotropic monomeric and exchange-coupled polynuclear $d$ and $f$-block complexes, J. Comput. Chem. 34, 1164 (2013).

[44] J. M. Alameda, D. Givord, R. Lemaire, and Q. Lu, Co energy and magnetization anisotropies in $\mathrm{RCo}_{5}$ intermetallics between 4.2 K and 300 K, J. Appl. Phys. 52, 2079 (1981).

[45] J. B. Staunton, L. Szunyogh, A. Buruzs, B. L. Gyorffy, S. Ostanin, and L. Udvardi, Temperature dependence of magnetic anisotropy: An ab initio approach, Phys. Rev. B 74, 144411 (2006).

[46] C. E. Patrick, S. Kumar, K. Götze, M. J. Pearce, J. Singleton, G. Rowlands, G. Balakrishnan, M. R. Lees, P. A. Goddard, and J. B. Staunton, Field-induced canting of magnetic moments in $\mathrm{GdCo}_{5}$ at finite temperature: First-principles calculations and high-field measurements, J. Phys.: Condens. Matter 30, 32LT01 (2018).

[47] A. V. Andreev, Thermal expansion anomalies and spontaneous magnetostriction in rare-earth intermetallics with cobalt and iron, in Handbook of Magnetic Materials (Elsevier NorthHolland, New York, 1995), Chap. 2, p. 59.

[48] K. H. J. Buschow, Intermetallic compounds of rare-earth and 3d transition metals, Rep. Prog. Phys. 40, 1179 (1977).

[49] E. Bruno and B. Ginatempo, Algorithms for Korringa-KohnRostoker electronic structure calculations in any Bravais lattice, Phys. Rev. B 55, 12946 (1997).

[50] S. H. Vosko, L. Wilk, and M. Nusair, Accurate spin-dependent electron liquid correlation energies for local spin density calculations: A critical analysis, Can. J. Phys. 58, 1200 (1980).

[51] O. Eriksson, B. Johansson, R. C. Albers, A. M. Boring, and M. S. S. Brooks, Orbital magnetism in $\mathrm{Fe}, \mathrm{Co}$, and $\mathrm{Ni}$, Phys. Rev. B 42, 2707 (1990).

[52] See Supplemental Material at http://link.aps.org/supplemental/ 10.1103/PhysRevMaterials.3.101401 for tables of DFT-DLM calculated parameters and CF coefficients, the experimental $\mathrm{RCo}_{5}$ lattice constants used, a description of the SucksmithThompson method used to extract anisotropy constants, and calculations testing the extent of canting in $\mathrm{PrCo}_{5}$ and $\mathrm{CeCo}_{5}$.

[53] J. Enkovaara, C. Rostgaard, J. J. Mortensen, J. Chen, M. Dułak, L. Ferrighi, J. Gavnholt, C. Glinsvad, V. Haikola, H. A. Hansen et al., Electronic structure calculations with GPAW:
A real-space implementation of the projector augmented-wave method, J. Phys.: Condens. Matter 22, 253202 (2010).

[54] R. J. Elliott, in Magnetic Properties of Rare Earth Metals (Plenum Press, New York, 1972), p. 2

[55] A. Arrott, Criterion for ferromagnetism from observations of magnetic isotherms, Phys. Rev. 108, 1394 (1957).

[56] R. J. Radwański, The origin of the basal-plane magnetocrystalline anisotropy in $4 f$ Co-rich intermetallics, J. Phys. F: Met. Phys. 17, 267 (1987).

[57] S. A. Nikitin, K. P. Skokov, Y. S. Koshkid'ko, Y. G. Pastushenkov, and T. I. Ivanova, Giant Rotating Magnetocaloric Effect in the Region of Spin-Reorientation Transition in the $\mathrm{NdCo}_{5}$ Single Crystal, Phys. Rev. Lett. 105, 137205 (2010).

[58] M. Ohkoshi, H. Kobayshi, T. Katayama, M. Hirano, T. Katayama, M. Hirano, and T. Tsushima, Spin reorientation in $\mathrm{NdCo}_{5}$ single crystals, AIP Conf. Proc. No. 29 (AIP, Melville, NY, 1976), p. 616.

[59] S. Kumar, C. E. Patrick, R. S. Edwards, G. Balakrishnan, M. R. Lees, and J. B. Staunton, Study of the spin reorientation transition and magnetocrystalline anisotropy in $\mathrm{NdCo}_{5}$ using torque magnetometry (unpublished).

[60] H. C. Herper, T. Ahmed, J. M. Wills, I. Di Marco, T. Björkman, D. Iuşan, A. V. Balatsky, and O. Eriksson, Combining electronic structure and many-body theory with large databases: A method for predicting the nature of $4 f$ states in Ce compounds, Phys. Rev. Mater. 1, 033802 (2017).

[61] M. J. Lipp, D. Jackson, H. Cynn, C. Aracne, W. J. Evans, and A. K. McMahan, Thermal Signatures of the Kondo Volume Collapse in Cerium, Phys. Rev. Lett. 101, 165703 (2008).

[62] E. Tatsumoto, T. Okamoto, H. Fujii, and C. Inoue, Saturation magnetic moment and crystalline anisotropy of single crystals of light rare earth cobalt compounds $\mathrm{RCo}_{5}$, J. Phys. Colloq. 32, C1 (1971).

[63] R. Radwański, The rare earth contribution to the magnetocrystalline anisotropy in $\mathrm{RCo}_{5}$ intermetallics, J. Magn. Magn. Mater. 62, 120 (1986).

[64] K. Buschow, A. van Diepen, and H. de Wijn, Crystal-field anisotropy of $\mathrm{Sm}^{3+}$ in $\mathrm{SmCo}_{5}$, Solid State Commun. 15, 903 (1974).

[65] M. Richter, Band structure theory of magnetism in 3d-4f compounds, J. Phys. D: Appl. Phys. 31, 1017 (1998). 